\title{
Reducing Preanalytical Laboratory Sample Errors Through Educational and Technological Interventions
}

\author{
ROSA LILLO ${ }^{1}$, MARÍA SALINAS ${ }^{1}$, MAITE LOPEZ-GARRIGÓS ${ }^{1}$, \\ YURENA NARANJO-SANTANA ${ }^{1}$, MERCEDES GUTIÉRREZ ${ }^{1}$, MARIA DOLORES MARÍN ${ }^{1}$, \\ MANUEL MIRALLES ${ }^{1}$, JOAQUÍN URIS ${ }^{2}$ \\ ${ }^{1}$ Clinical Laboratory Department, University Hospital of San Juan, Alicante, Spain \\ ${ }^{2}$ Department of Public Health, University of Alicante, Alicante, Spain
}

\begin{abstract}
SUMMARY
Background: A correct preanalytical phase procedure is critical to get an adequate sample and consequently to achieve the most reliable laboratory results, promoting patient safety. Continuous laboratory staff changes create the need to establish improvement strategies to reduce the error risk. The objective was to show how the numbers of preanalytical errors related to unsuitable samples in a hospital setting decrease following two improvement strategies related to new technology and educational actions and how their effects were measured by monitoring indicators.

Methods: Samples were drawn by the laboratory and other hospital departments' nurses without previous patient appointment, therefore, prior tube preparation was not available before the phlebotomy. Corrective measures for these activities were established: educational program for nurses and a system of custom labels, which correlate each laboratory test in the Laboratory Information System (LIS) with the corresponding tube. Three phases were defined based on the implementation dates of the improvement actions to be assessed. The set of indicators designed to monitor the improvement related to clotted, hemolyzed, insufficient, and uncollected samples. Data were collected and indicators calculated from the LIS using a data warehouse application. Patient satisfaction with respect to phlebotomy was also measured annually using a scoring survey.

Results: There was a reduction in all types of preanalytical sample errors related to the improvement strategies adopted. The indicators demonstrated that the unavailable, insufficient, and clotted samples decreased between two- and three-fold, whereas hemolysis errors benefited more from these improvement strategies.

Patient satisfaction with the laboratory and phlebotomy procedures improved over the past several years as based on the annual satisfaction surveys.

Conclusions: The educational program for nursing personnel is relevant and important as can be seen in the decrease of sample errors and the resulting quality improvement. The custom label system minimizes the potential oversight of forgetting to draw a tube, which happens frequently when operating without appointments, by print ing the labels according to requested tests. Detection, identification, and monitoring of the error and implementing strategies to improve preanalytical quality reduces error numbers and thereby improves patient safety and health system outcomes.
\end{abstract}

(Clin. Lab. 2012;58:xx-xx. DOI: 10.7754/Clin.Lab.2012.111111)

\section{KEY WORDS}

Improvement strategies, laboratory errors, patient safety, preanalytical phase, phlebotomy, quality in laboratory

Manuscript accepted January 14, 2012

\section{INTRODUCTION}

Clinical laboratory sample analysis is very important in health care, as it leads to results that affect clinical aspects regarding the patient's diagnosis, treatment or health status follow up. The laboratory report has an influence on $60-70 \%$ of clinical decisions made. [1] 
The clinical laboratory's main objective should be delivering quality results achieved through an appropriate and standardized sample treatment throughout the entire process, from the beginning of sample collection to the delivery and communication of the laboratory report. The daily laboratory professional challenge must be to minimize the possible errors at any phase during this process. Therefore corrective measures must be applied, if necessary, and subsequent evaluation of the improvement is essential.

The analytical process is a set of consecutive tasks, improving each phase to be as effective as possible to enhance the quality and efficiency in the organization.

Nowadays, the testing process still requires extensive manual management through the different laboratory procedures (pre-, intra- and post-analytical) which carries a percentage of error risk. A laboratory error was defined by the International Organization for Standardization (ISO) as "any failure of planned action to be completed as intended, or use a wrong plan to achieve an aim, occurring at any part of the laboratory cycle, from ordering examinations to reporting results and appropriately interpreting and reacting to them" [2]. The main laboratory error source is the preanalytical phase, where the phlebotomy and subsequent sample distribution are carried out, actions that are fundamentally manual. "According to reliable data, preanalytical errors still account for nearly $60-70 \%$ of all problems occurring in laboratory, most of them attributable to mishandling procedures during collection, handling, preparing or storing the specimens" [3]. Substantial advances in technology, automation, and analytical quality over time has improved laboratory performance, while at the same time has increased the absolute amount of laboratory reports. However, automation implementation is not expected to exert a substantial influence on phlebotomy errors. It is necessary to establish different strategies to prevent the weakness in this crucial procedure. Frequent turnover of the nurses performing phlebotomies and "shortages of skilled staff and overloaded systems create new occasions for basic but easily preventable errors that might compromise patient safety and introduce unjustified health expenditure" [4].

Other issues that must be taken into account to ensure proper collecting of patient samples is the patient identification and number of samples to be drawn. "The preanalytic phase enfolds the greatest potential for quality improvement, once reliable strategies are identified and applied" [5]. The laboratory strategy must begin by creating an error identification policy, monitored with indicators [6], and the development of improvement strategies for their reduction [7].

The study objective was to show how the number of preanalytical errors related to unsuitable samples in a hospital setting decrease following two improvement strategies established usig new technology and education, and how their effects were measured by monitoring with indicators.

\section{MATERIALS AND METHODS}

The study was carried out in the Laboratory of the San Juan Hospital in Alicante, which serves a population of 234551 inhabitants and is highly targeted to oncology patients. The laboratory attends in and outpatients and also receives primary care patient samples obtained in Primary Care Centers, which are transported to the hospital laboratory.

This study focuses on outpatients' samples that were drawn by the laboratory nurses and supported by nurses from other hospital departments.

Outpatients' samples were obtained without previous patient appointment so prior preparation of the tubes was not possible before the phlebotomy representing a possible source of error in the sequential preanalytical process.

The impact of two improvement actions aimed at reducing the amount of errors in our hospital/laboratory were measured.

The study period was established from July 2007 - July 2010 and three phases were defined (Aug 2007 - Jan 2008; Feb 2008 - Oct 2009; and Nov 2009 - Jul 2010), based on the dates of implementation of the improvement actions, in order to evaluate their effectiveness. In January 2008, a system of custom labels was established for each patient request. Each laboratory test in the Laboratory Information System (LIS) was associated with a corresponding tube. When a request was registered, the tube's identifying labels were printed in accordance with the tests requested. In October 2009, a host plan was set up for nursing personnel attending the laboratory for the first time. This consisted of an informative leaflet (Figure 1) describing the laboratory sample drawing procedure and encouraging them to ask for help from coworkers, if needed. The host plan was explained by the laboratory nurse supervisor to each nurse on the first day of work, clearing any doubts, and assigning an expert coworker to monitor the new nurse daily during the first month.

A sample error was defined as a rejected specimen: any blood or urine sample which cannot be successfully tested as it does not meet the laboratory acceptability criteria or if the sample is not collected [8].

Preanalytical errors were seen in tests requested for hematology (EDTA tube), coagulation (citrate tube), chemistry (serum tube), and urine samples. Specimens that were clotted, insufficient, hemolyzed or uncollected (sample needed for processing requested tests, but not collected) were included.

In the hematology and coagulation samples, clotted, insufficient, and uncollected samples were seen as errors. In the chemistry samples, errors were hemolyzed and uncollected samples and in the urine samples, the only error considered was uncollected.

When an unsuitable sample is identified, a specificly coded result was registered in the LIS as the test result in order to report the incident and to request a new sample. Such coded results (preanalytical errors) were iden- 
1. VALENCIANA DE SALUT

\section{Service of Clinical Analysis Hospital of San Juan}

$\checkmark$ The patient should be called by name and first surname and to confirm his/her identity we need to ask for his/her second surname. Be sure to identify correctly the patient.

$\checkmark$ Check that the identification number from the request form and the tube labels matches.

$\checkmark$ Check that the tubes prepared and labeled by the lab assistant correspond to those necessary for the requested tests. The necessary tubes for the different tests are found in the request form; the codes are consulted on the underside.

$\checkmark$ If the request form has a small label stuck on the edge, solicit urine, feces or/and sputum from the patient according to the request

$\checkmark$ Before starting the phlebotomy inform the patient about the technique you will perform. This help the patient assume the proper position and transmits safety and calm.

$\checkmark$ Fill the tubes following the order from the table below and once filled make the inversions needed for each tube:

\begin{tabular}{|c|c|c|}
\hline $\mathbb{0}$ & Tube with gel separator and coagulation activator & $\begin{array}{c}6 \\
\text { inversions }\end{array}$ \\
\hline 17 & Citrate tube & $\begin{array}{c}\text { 3/4 } \\
\text { inversions }\end{array}$ \\
\hline & Heparin tube & $\begin{array}{c}8 / 10 \\
\text { inversions }\end{array}$ \\
\hline$[1$ & EDTA tube & $\begin{array}{c}8 / 10 \\
\text { inversions }\end{array}$ \\
\hline 411 & Other tubes with additives: fluoride... & $\begin{array}{c}8 / 10 \\
\text { inversions }\end{array}$ \\
\hline
\end{tabular}

$\checkmark$ Write your initials on the request form.

$\checkmark$ Arterial Blood Gases and cold samples (underside*) require immediate transfer to the laboratory by the phlebotomy lab assistant.

A good phlebotomy is critical for the quality of the sample and that of the results. Remember:

$\checkmark \quad$ If the phlebotomy is with the traditional syringe-needle method, do not aspirate too strongly to avoid hemolysis

$\checkmark \quad$ If it is with the vacuum method, give the tubes the necessary time to fill them till their volume is adequate.

\section{IF YOU HAVE TRIED THE PATIENT PHLEBOTOMY ONCE WITHOUT} SUCCESS, ASK FOR ASSISTANCE!

Figure 1. Informative leaflet of the host plan at the Clinical Laboratory for nursing professionals.

The figure shows the informative leaflet, integrated into the host plan, which is given to the nursing professionals upon their arrival to the laboratory. 
2A. Hematology

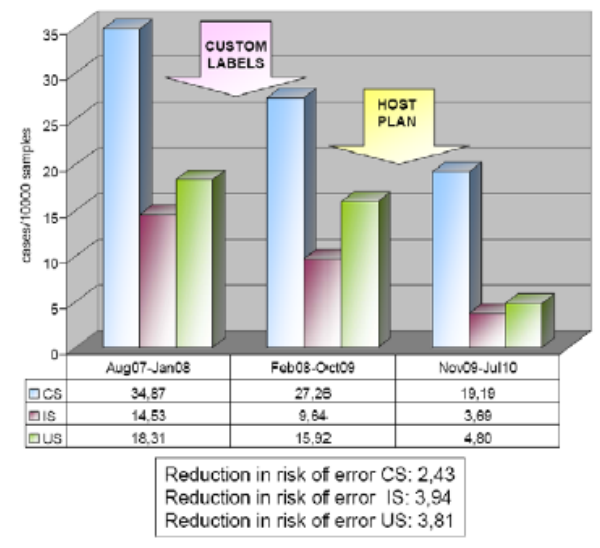

2C. Chemistry

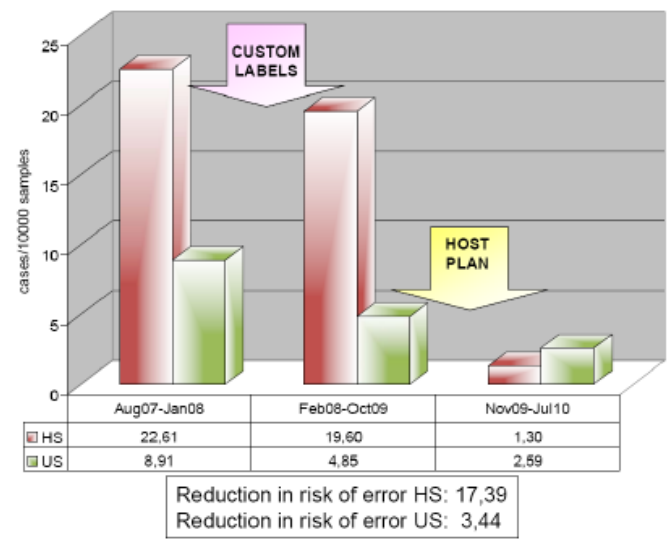

2B. Coagulation

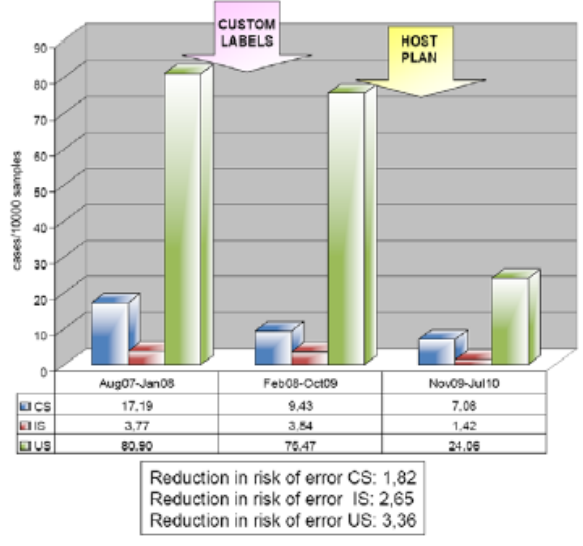

2D. Urine

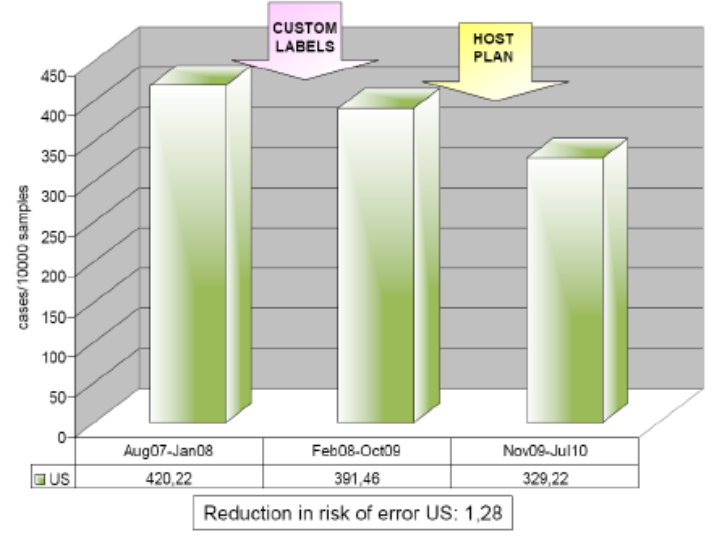

Figure 2. Evolution of errors by periods studied and improvement actions (cases/10000 samples).

The figure shows the errors in each sample type, expressed in cases/10000 samples during the periods studied in accordance with the improvement actions implemented.

Abbreviations: CS: clotted sample; IS: insufficient sample; US: unavailable sample; HS: hemolyzed sample.

tified and collected automatically from the LIS.

To establish the improvement strategies, monthly indicators were designed and classified as individual and global. Individual indicators represent each type of preanalytical error with respect to every sample collected (three in hematology and coagulation, two in chemistry, and one in urine samples). Global indicators represent the sum of all types of preanalytical errors with respect to every sample collected (there are three; urine is the same for global and individual).

The indicators were collected and calculated using a software program based on Data Warehouse and OnLine Analytical Processing (OLAP) cube (Data Ware- house Omnium ${ }^{\circledR}$ Roche Diagnostics). The number of errors per 10000 samples was calculated. The decrease in the risk of error was also calculated by dividing the number of errors in the first period (preceding the improvement actions) by the number of errors that occurred in the last period. The graphic representation of the indicator results is shown in Microsoft Excel 2003®.

Patient satisfaction with respect to phlebotomy was measured annually through customer satisfaction surveys, which were evaluated on a scale of 0 to $10(0$, poor and 10, excellent). The survey contains 16 questions 6 refer to the phlebotomy room (cleanliness, com- 


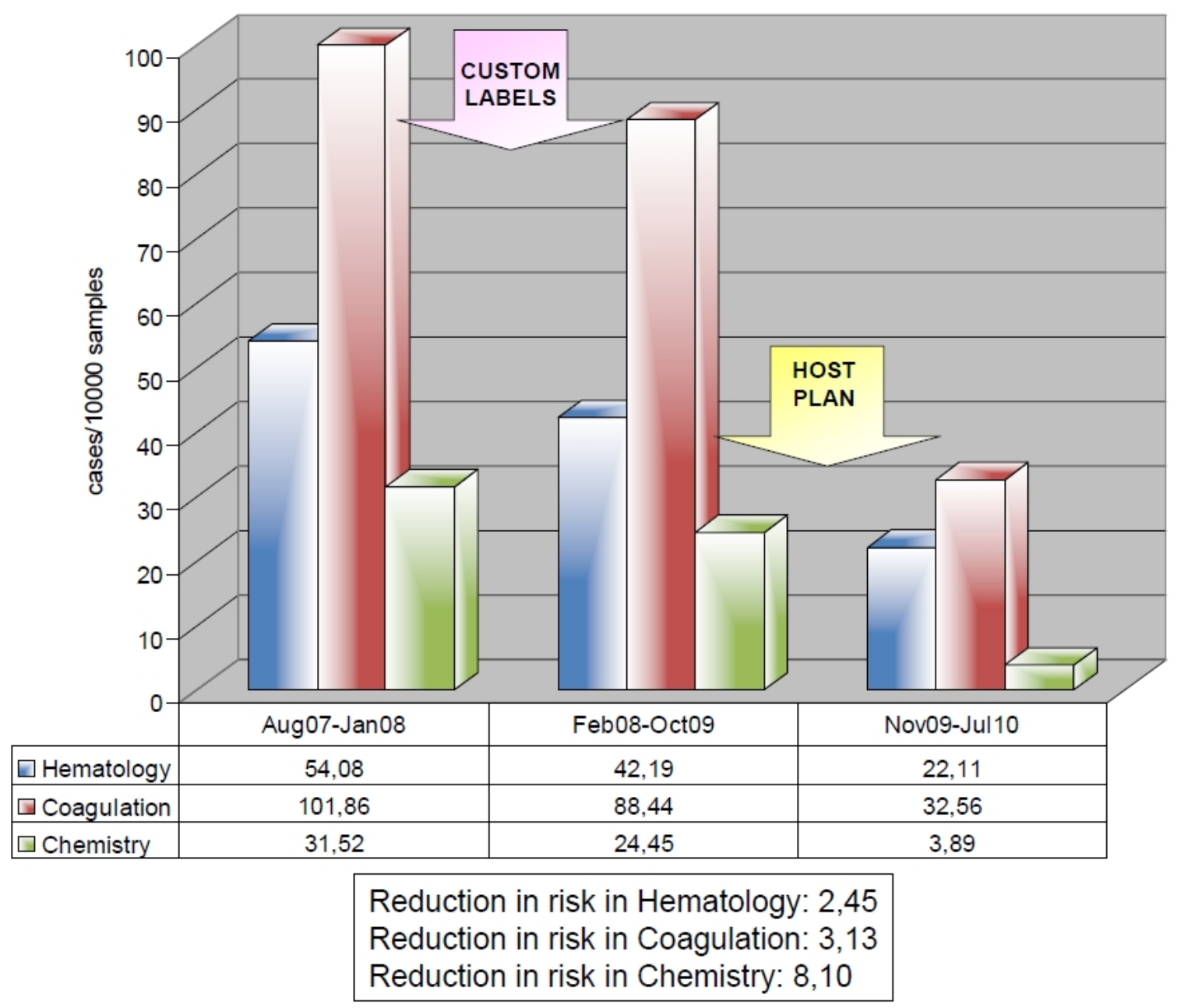

Figure 3. Evolution of errors as a function of each sample type according to periods studied and improvement actions (cases/10000 samples).

The figure shows the errors for each type of sample expressed in cases/10000 samples during the periods studied in accordance with the improvement actions implemented.

Abbreviations: CS: clotted sample; IS: insufficient sample; US: unavailable sample; HS: hemolyzed sample.

fort ...), 6 to the drawing procedure, 3 refer to the laboratory administrative staff that registers the patient's data, and finally one question evaluates the overall service.

During the study period, there were no other technological or procedural changes for the shown improvements.

\section{RESULTS}

During the study period (from July 2007 - July 2010) 136,479 requests and 2,296,695 tests were received. In the first phase (Aug 2007 - Jan 2008), 16602 theoretical hematology, 5300 coagulation, 12383 chemistry, and 2248 urine samples were registered. In the second phase (Feb 2008 - Oct 2009), 60574, 19777, 44896, and 8686 samples, respectively, were registered as well; during the third phase (Nov 2009 - Jul 2010), 21708, 7067,15444 , and 2673, respectively, were registered. 
The most frequent error in hematology tubes was a clotted sample, while for coagulation and chemistry it was an unavailable sample. The risk of committing each type of error for each type of sample diminished following the implementation of the improvement actions as evidenced in the study evolution.

The results are graphically shown (Figure 2) in four graphics, one for each type of sample: samples of hematology (Figure 2A), coagulation (Figure 2B), chemistry (Figure 2C), and urine (Figure 2D). In all graphics the baseline for laboratory preanalytical errors can be seen and then the decrease with the first improvement action (custom labels) and finally the effect of both (custom labels and host plan) following the establishment of the host plan.

Preanalytical error evolution is observed and expressed in cases per 10000 samples. Figure 3 also shows the improvement in the global indicators after the two interventions. The risk of committing an error after implementing the improvement actions diminished in all cases, reaching 8.16 times for the chemistry sample. Patient satisfaction with laboratory attendance and phlebotomy procedure has improved over the past several years (average scores 7.9, 8, 8.3, and 8.3 since 2007). Annual results (average score) since 2007 are shown.

\section{DISCUSSION}

Three basic pillars exist for improving patient safety from the laboratory. The first one is the improvement in the clinician-laboratory professional interface in order to obtain improvements in test requests as much as in report interpretation, i.e. redirecting the laboratory towards a customer service team. Second is the continuous improvement in analytical quality; even though few errors are described in this stage, these are the ones that carry the highest risk [9]. Lastly, improving preanalytical quality, because this is where most errors are described [3] but at the same time, this is where they are most easily avoidable. Hence, laboratories must be encouraged focus on continuous improvement in preanalytical quality [10].

Some errors may be due to poor communication, action taken by others involved in the testing process (e.g., physicians, nurses, and phlebotomists), or a poorly designed-process, all which are beyond the laboratory's control [1]. Preanalytical errors caused by unsuitable samples resulting from defective sampling procedure can be serious and cause an inadequate result in diagnosis, treatment or prevention [11]. These errors can also be null or minor and provoke diagnostic delay or patient discomfort, like a second phlebotomy [3], creating the need to establish appropriate measures for their reduction. It is necessary to detect, identify, and monitor the error in an automated and continuous manner over time [1]. Standardization and monitoring preanalytical variables is of foremost importance and is associated with the most efficient and well-organized laboratories, re- sulting in reduced operational costs and increased revenues [12].

It is important to be aware of any changes that may increase laboratory errors and accordingly establish corrective measures trying to increase patient safety. Although human conditions cannot be changed, the conditions under which humans work and can be controled and can be improved upon [2]. Historically, phlebotomy was always performed by laboratory personnel. However, the gradual arrival of laboratory technicians has led to progressive disappearance of laboratory nurses responsible for sampling procedures. Phlebotomy success, a critical prerequisite for suitable specimens, is dependent upon the degree of laboratory supervision of this procedure [4]. This created the need for corrective measures to be applied.

The fact that patients do not have appointments for the laboratory does not allow for prior LIS patient registration or prior preparation of the tubes that nurses have to draw for each patient. This is a clear cause of the "not collected" samples due to personnel.

The custom label system has managed to avoid missing samples by printing labels according to the requested tests.

The big difference between IS and US in coagulation samples as compared with the same errors in haematology, could be explained by the limited coagulation tests requested that leads to forgetfulness. However, complete blood count is requested almost systematically in every patient.

The study makes use of daily testing laboratory registers that are indispensable in providing unsuitable test specimen results to construct the indicators. The collection of the LIS registers and the calculation of the indicators are carried out automatically and in real time with a computer application based on Data Warehouse. This implies three great advantages: there are no register losses and there is continuous collection and acquisition of the preanalytical quality indicators in real time without any additional effort.

The study results demonstrate that the two improvement actions implemented in the laboratory have reduced the number of errors in the two periods of the study. The various indicators studied show how the unavailable, insufficient, and clotted samples decreased between two and three times, whereas errors from hemolysis improved by a much greater proportion, and this is the error that benefits more from these improvement actions.

The sample drawing procedure is especially sensitive to technical problems; it is the only moment the patient is in contact with the laboratory personnel, and as such, it requires special attention. The training, ability, and implication of the professionals is fundamental. As the study results show, the host plan for nursing personnel is both relevant and very important with regards to the decrease in errors and the resulting quality improvement. Patients have become more satisfied with attendance laboratory and phlebotomy procedure every year, as can be seen in the annual satisfaction surveys. 
The issuance of custom labels minimizes the potential oversight of forgetting to draw a sample, which happens frequently when we are operating without appointments where neither prior LIS patient registration nor prior preparation of the tubes is possible.

It is very difficult to answer whether it is worth more to improve technical circumstances or staff training to minimize particular errors based on the study data. But what is certain is that the drawing procedure is the only moment the patient contacts the laboratory and it is therefore essential to establish the maximum number of improvement strategies

The laboratory is involved in many medical procedures, and therefore small efforts can lead to large results. Laboratory professionals, as demonstrated in this study, must take advantage of all the tools available within their reach $[13,14]$, whether they are technological or training [15] in order to identify and diminish the error [16] throughout the entire process and promote continuous improvement which will always benefit patient safety [3].

\section{Acknowledgements;}

The authors wish to thank all laboratory personnel who contributed to the delivery of laboratory services.

\section{Declaration of Interest:}

None conflicts declared.

\section{References:}

1. Forsman RW. Why is the laboratory an afterthought for managed care organizations? Clin Chem 1996;42:813-6.

2. International Organisation for Standardisation/Technical Specification. Medical laboratories - reduction of error through risk management and continual improvement. ISO/TS 22367:2008.

3. Lippi G, Chance JJ, Church S, et al. Preanalytical quality improvement: from dream to reality. Clin Chem Lab Med 2011; 49:1113-26.

4. Lippi G, Salvagno GL, Franchini M, Guidi GC. Phlebotomy Issues and Quality Improvement in results of laboratory testing. Clin Lab 2006;52:217-30.
5. Lippi G, Guidi GC. Preanalytic indicators of laboratory performances and quality improvement of laboratory testing. Clin Lab 2006;52:457-62.

6. Salinas M, López-Garrigós M, Gutiérrez M, Lugo J, Sirvent JV Uris J. Achieving continuous improvement in laboratory organization through performance measurements: a seven-year experience. Clin Chem Lab Med_2010;48:57-61.

7. Signori C, Ceriotti F, Sanna A, et al. Process and risk analysis to reduce errors in clinical laboratories. Clin Chem Lab Med 2007; 45:742-8.

8. Jones BA, Calam RR, Howanitz PJ. Chemistry specimen acceptability: a College of American Pathologists Q-Probes study of 453 laboratories. Arch Pathol Lab Med 1997;121:19-26.

9. Salinas M, Lopez-Garrigos M, Flores E, Gutiérrez M, Lugo J, Uris J. Three years of preanalytical errors: quality specifications and improvement through implementation of statistical process control. Scand J Clin Lab Invest 2009;69:822-6.

10. Söderberg J, Wallin O, Grankvist K, Brulin C. Is the test result correct? A questionnaire study of blood collection practices in primary health care. Eval Clin Pract 2010;16:707-11.

11. Emons MF. Integrated Patients data for optimal patient management: the value of laboratory data in quality improvement. Clin Chem 2001;47:1516-20.

12. Goswami B, Singh B, Chawla R, Mallika V. Evaluation of errors in a clinical laboratory: a one-year experience. Clin Chem Lab Med 2010;48:63-6.

13. Bell D, McNaney N, Jones M. Improving health care through redesign. BMJ 2006;332:1328-30.

14. Lippi G, Banfi G, Buttarello M, et al. Recommendations for detection and management of unsuitable samples in clinical laboratories. Clin Chem Lab Med 2007;45:728-36

15. Lippi G, Guidi GC. Risk management in the preanalytical phase of laboratory testing. Clin Chem Lab Med 2007;45:720-7.

16. Boone DJ. How can we make laboratory testing safer? Clin Chem Lab Med 2007;45:708-11.

\section{Correspondence:}

Maite López-Garrigós

Carretera Alicante-Valencia, s/n

03550 Alicante, Spain

Tel.: $\quad+34-96-5938877$

Fax: $\quad+34-96-5938683$

Email: lopez_marter@gva.es 